\title{
Gender-wise Variation in Performance in Formative Assessment and Class Attendance in the Subject of Biochemistry in First-year MBBS Students
}

\author{
Pratik Raghavani ${ }^{1}$, Ajay S Rajput ${ }^{2}$, Deepak Parchwani ${ }^{3}$, Uday Vachhani $^{4}$
}

\begin{abstract}
Introduction: The medical profession is one of the most demanding disciplines and historically remained a male-dominated profession. Academic performance in medical science is affected by various sociodemographic factors. Various studies have been done to predict academic performance in medical school regarding the influence of gender and attendance. In this study, an attempt was made to study the effect of gender and attendance on performance in formative assessment in the subject of biochemistry.

Aims and objectives: To compare performance in theory, practical, and combined (theory and practical) formative assessments between male and female students in the subject of biochemistry in the first-year MBBS and to compare class attendance in theory, practical, and combined (theory and practical) between male and female students in the subject of biochemistry in the first-year MBBS.

Materials and methods: This study was carried out at the Department of Biochemistry, GMERS Medical College, Himmatnagar. Students' academic records in the subject of biochemistry during their first professional year of MBBS were studied. For the study, data of 450 ( 251 male and 198 female) students from 3 academic years were collected. Performance in theory and practical formative assessments was compared between male and female students. A comparison of marks of male and female students was carried out using Student's $t$-test. The effect of attendance on performance was assessed gender-wise. For comparison of attendance, Kruskal-Wallis test was applied. For all comparisons, a $p$ value $<0.05$ was considered significant. All statistical analyzes were carried out using Microsoft Excel 2010.

Results: The performance of female students was better than male students in theory and combined formative assessments $(p<0.05)$. The attendance of female students in theory classes and combined attendance of theory and practical was significantly higher $(<0.05)$ than their male counterparts. In different attendance groups for both male and female students, the performance was significantly better with more attendance $(p<0.05)$.

Discussion: In our study, we explored how gender and attendance can affect the performance of the student in the subject of biochemistry. Our study demonstrates female students have more class attendance and better performance in formative assessment in the subject of biochemistry and both the genders' performances are positively correlated with their attendance.

Keywords: Female, Gender, Male, Performance, Practical, Theory.

Indian Journal of Medical Biochemistry (2020): 10.5005/jp-journals-10054-0159
\end{abstract}

\section{INTRODUCTION}

Medical education is one of the most demanding disciplines in which students experience various stressors such as academic workload, lack of recreational time, competition, emotional pressure, financial hardship, the pressure to succeed, and worries about the future. ${ }^{1,2}$ Considering all these factors and the conservative nature of Indian families, medicine remained a male-dominated profession for a long period in India. In 1885, to deliver scholarships, to educate women in the medical field, The Countess of Dufferin Fund was established. ${ }^{3}$ In 1886, Dr Anandibai Joshi became the first Indian woman to qualify as a doctor. Since then there has been a constant rise in the number of females entering the medical profession, though exact data on a nationwide basis are not available. ${ }^{4}$

Academic performance is affected by various sociodemographic factors like the medium of instruction in higher secondary studies, previous academic performance, and socioeconomic status, educational qualification of parents, class attendance, and gender. ${ }^{5,6}$ Several studies have been done to predict academic performance in medical school regarding the influence of gender. Some studies found that women performed better in academics than men. ${ }^{7-10}$ Faisal et al. do not found a significant difference in academic performance. ${ }^{11}$
${ }^{1-4}$ Department of Biochemistry, Gujarat Medical Education and Research Society Medical College, Himmatnagar, Gujarat, India

Corresponding Author: Ajay S Rajput, Department of Biochemistry, Gujarat Medical Education and Research Society Medical College, Himmatnagar, Gujarat, India, Phone: +91 9924060088, e-mail: ajayrajput85@gmail.com

How to cite this article: Raghavani P, Rajput AS, Parchwani D, et al. Gender-wise Variation in Performance in Formative Assessment and Class Attendance in the Subject of Biochemistry in First-year MBBS Students. Indian J Med Biochem 2020;24(3):92-95.

Source of support: Nil

Conflict of interest: None

Biochemistry is one of the basic science subjects in the first professional year of MBBS. Proper knowledge of basic science subjects is very important for clinical decision-making skills. The effect of gender on performance in clinical subjects like surgery, medicine, pediatrics, psychiatry, and obstetrics, and gynecology has been studied but the same is not studied extensively in the subject of biochemistry. ${ }^{7}$ In this study, an attempt was made to study the 
effect of gender and attendance on performance in formative assessment in the subject of biochemistry.

\section{Aims and Objectives}

- To compare performance in theory, practical, and combined (theory and practical) formative assessments between male and female students in the subject of biochemistry in the firstyear MBBS.

- To compare class attendance in theory, practical, and combined (theory and practical) between male and female students in the subject of biochemistry in the first-year MBBS.

\section{Materials and Methods}

This study was carried out at the Department of Biochemistry, GMERS Medical College, Himmatnagar after approval by the Institutional Ethics Committee GMERS Medical College, Gandhinagar. Students' academic records in the subject of Biochemistry during their 1st professional year of MBBS were studied. For the study, data of 450 students of 3 academic years viz. 2015 to 2016 (n-150), 2016 to 2017 ( $n-150)$, and 2017 to 2018 ( $n-150)$ were collected. Students who had taken at least five formative assessments were included in the study. Students not fitting into the study protocol were excluded. In the study, one male student was excluded because he did not comply with the study protocol. To assess performance in formative assessment total of marks of periodic theory examinations, periodic practical examinations, viva-voce, term ending examination (theory and practical), and preliminary examination (theory and practical) were considered and these records were segregated gender-wise. As the sum of maximum marks in all formative assessments was different for all three academic years, they were expressed as a percentage, to make the data comparable. For that, obtained marks of all the theory formative assessments were sum up and were expressed as a percentage of the sum of maximum marks of all the theory formative assessments. In the same manner, percentage marks for practical assessments and combined assessments were also derived. For combined assessments, theory and practical assessments were given one: one weightage. Performance in theory and practical formative assessments was compared between male and female students. Performance in combined (theory and practical) formative assessments were also compared. A comparison of marks of male and female students was carried out using Student's $t$-test and $p$ value $<0.05$ was considered significant. Gender-wise segregation of attendance records of theory and practical classes was done. Attendance of theory classes, practical classes, and total attendance (theory and practical combined) was compared between male and female students.

To know the effect of attendance on performance, male students were grouped into three categories: (1) $<80 \%$, (2) between 80 and $90 \%$, and (3) $>90 \%$. Similarly, the effect of attendance on performance was assessed also for female students. For comparison of attendance, Kruskal-Wallis test was applied. All statistical analyzes were carried out using Microsoft Excel 2010.

\section{Results}

The study population consisted of 251 male students and 198 female students. The mean and standard deviation of percentage marks in theory, practical, and combined formative assessments were calculated for male and female students. Results of the Student's $t$-test showed the performance of female students was better than males in theory and combined formative assessments $(p<0.05)$ while for practical formative assessments no significant difference was found (Table 1). For comparison of attendance, median and interquartile range of attendance records of male and female students were calculated for theory, practical, and combined classes. Results from the Kruskal-Wallis test show that the attendance of female students in theory classes and combined attendance of theory and practical was significantly higher $(<0.05)$ than their male counterparts (Table 2). One-factor ANOVA revealed a significant difference $(<0.05)$ in the performance, in all the three categories of attendance for both male and female students (Table 3).

Table 1: Marks comparison between male and female

\begin{tabular}{lllll}
\hline & & Mean & SD & pvalue \\
\hline Theory & Male $(n=251)$ & 34.75 & 13.48 & $<0.05$ \\
& Female $(n=198)$ & 39.17 & 13.92 & \\
Practical & Male $(n=251)$ & 50.09 & 12.41 & 0.374262 \\
& Female $(n=198)$ & 51.13 & 11.93 & \\
Combined (theory and practical) & Male $(n=251)$ & 42.42 & 11.93 & $<0.05$ \\
& Female $(n=198)$ & 45.15 & 11.51 & \\
\hline
\end{tabular}

Table 2: Attendance comparison between male and female

\begin{tabular}{lllll}
\hline & & Male $(n=251)$ & Female $(n=198)$ & p value \\
\hline Theory & Median & 80.15 & 82.44 & $<0.05$ \\
& Q1 & 76.03 & 77.44 & \\
& Q3 & 84.68 & 87.90 & \\
Practical & Median & 78.35 & 80.22 & 0.1995 \\
& Q1 & 74.29 & 75.00 & \\
Combined (theory and practical) & Q3 & 84.92 & 86.36 & $<0.05$ \\
& Median & 79.83 & 81.48 & \\
& Q1 & 75.35 & 76.42 & \\
& Q3 & 83.02 & 85.49 & \\
\hline
\end{tabular}




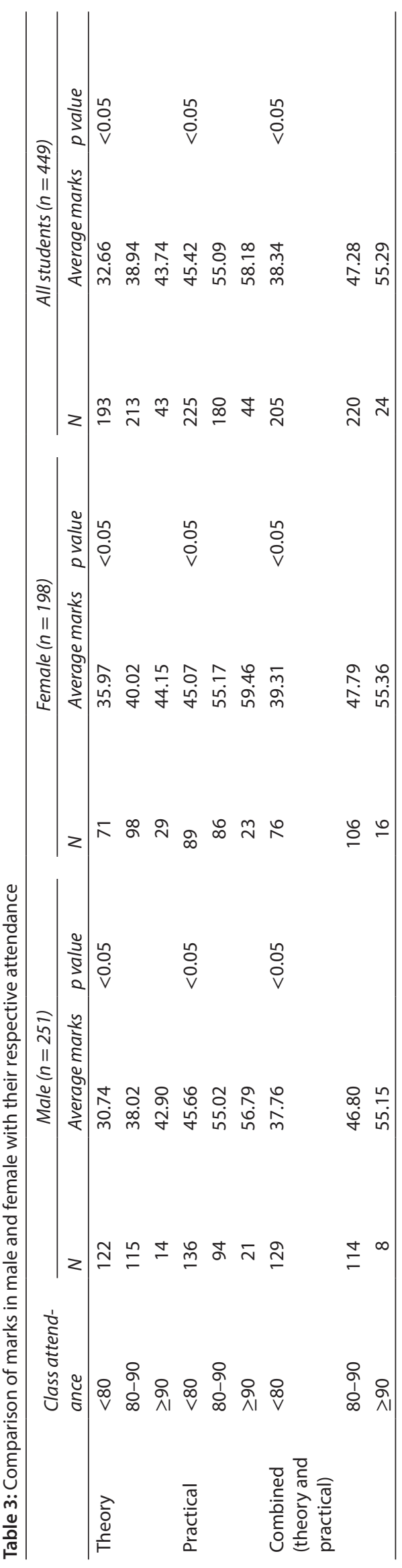

\section{Discussion}

The difference in academic performance can be attributed to multiple factors. ${ }^{5}$ In our study, we explored how gender and attendance can affect the performance of the student in the subject of biochemistry. At university college Dublin, McDonough et al. conducted a similar study for the final medical examination. They also found that females perform better than their male counterparts, especially in pediatrics, obstetrics and gynecology, and psychiatry. ${ }^{12}$ Bora and Baisya also reported a higher passing rate of female students in the first professional year of MBBS at Gauhati medical college. ${ }^{1}$ While another study carried out by Faisal et al. in the subject of pharmacology, showed no significant difference in the academic performance of female and male students. ${ }^{11}$ The current study showed female students performed better in formative assessments in the subject of biochemistry. This difference was due to their better performance in theory formative assessments as there was no significant difference in the performance of practical formative assessments. Furthermore, we studied attendance patterns gender-wise and found that female students were attending more theory classes than male students, but attendance in practical classes was not significantly different for male and female students. This suggests that the better performance of females in theory assessments may be due to their more attendance in theory classes and may not be attributed to their gender. To verify this, we assessed performance in different attendance groups for both males and females separately and found that students with more attendance performed better. However, Kauffman et al. found that classroom attendance is not significantly affecting performance which is contradictory to our findings. ${ }^{13}$ While some other studies reported a positive correlation of attendance on performance. Varu et al. conducted a study on 100 students and found a significant positive effect of attendance on performance in the subject of physiology. ${ }^{14}$ Similarly, findings were also reported by Mohanan et al. in the subject of pharmacology. ${ }^{15}$

\section{ConClusion}

Our study demonstrates female students have more class attendance and better performance in formative assessment in the subject of biochemistry and irrespective of gender, academic performance can be improved by attending more classes.

\section{ACKnOWLedgments}

We would like to show our gratitude to Dr Darshan Dave, Professor and Head, Department of Pharmacology, GMERS Medical College, Gandhinagar, Dr Rima Shah, Assistant Professor, Department of Pharmacology, GMERS Medical College, Gandhinagar, Dr Jaimin Patel, Assistant Professor, Department of Community Medicine, GMERS Medical College, Himmatnagar, Dr Sanjay Kanani, Assistant Professor, Department of Anatomy, GMERS Medical College, Gandhinagar, and Dr Nitish Tank, Tutor, Department of Pharmacology, GMERS Medical College, Himmatnagar for sharing their pearls of wisdom with us during this research.

\section{References}

1. Bora B, Baisya S. Gender variation in the result of first professional MBBS examination among the students of Gauhati Medical College. Indian J Basic App Med Res 2017;7(1):394-399.

2. Zeppegno P, Gramaglia C, Antona M, et al. Psychopathology, personality and theory of mind in a sample of university students. Riv Psichiatr 2014;49(3):132-139. 
3. Cooper H. The countess of Dufferin's fund, the national association for supplying female medical aid to the women of India. Hospital (Lond 1886) 1889;6(137):81-82.

4. Sood M, Chadda SK. Women in medicine: a perspective. Indian J Gender Stud 2010;17(2):277-285. DOI: 10.1177/097152151001700204.

5. Frischenschlager $\mathrm{O}$, Haidinger $\mathrm{G}$, Mitterauer L. Factors associated with academic success at Vienna Medical School: prospective survey. Croat Med J 2005;46(1):58-65.

6. Ekwochi U, Osuorah DC, Ohayi SA, et al. Determinants of academic performance in medical students: evidence from a medical school in south-east Nigeria. Adv Med Educ Pract 2019;10:737-747. DOI: 10.2147/AMEP.S210557.

7. Deepak KK, Al-Umran KU, Al-Sheikh MH, et al. The influence of gender on undergraduate performance in multiple choice testing in clinical disciplines at university of Dammam, Saudi Arabia. Al Ameen J Med Sci 2011;4(2):123-130.

8. Al-Mously N, Salem R, Al-Hamdan N. The impact of gender and english language on the academic performance of students: an experience from new Saudi Medical School. J Contemp Med Edu 2013;1(3):170-176. DOI: 10.5455/jcme.20130226121358.

9. Sawair FA, Baqain ZH, Kh, et al. Effect of gender on performance of undergraduate dental students at the University of Jordan, Amman. J Dent Educ 2009;73(11):1313-1319. DOI: 10.1002/j.00220337.2009.73.11.tb04823.x.
10. Katherine $\mathrm{W}$, Inam $\mathrm{H}$, Chris $\mathrm{Ml}$, et al. Exploring the underperformance of male and minority ethnic medical students in first year clinical examinations. Adv Health Sci Educat Theory Pract 2008;13(5):607-616. DOI: 10.1007/s10459-007-9067-1.

11. Faisal R, Shinwari L, Hussain SS. Academic performance of male in comparison with female undergraduate medical students in pharmacology examinations. J Pak Med Assoc 2017;67(2): 204-208.

12. McDonough $\mathrm{CM}$, Horgan $\mathrm{A}$, Codd MB, et al. Gender differences in the results of the final medical examination at University College Dublin. Med Educ 2000;34(1):30-34. DOI: 10.1046/j.1365-2923.2000. 00456.x.

13. Kauffman CA, Derazin M, Asmar A, et al. Relationship between classroom attendance and examination performance in a secondyear medical pathophysiology class. Adv Physiol Educ 2018;42(4):593598. DOI: 10.1152/advan.00123.2018.

14. Varu M, Vegad A, Shah C, et al. Attendance, attitudes and academic performance: a study on first year MBBS students attending physiology classes. IJMSE 2016;3(1):31-37.

15. Mohanan LK, Harichandran DT, Vijayan SM. Association of class attendance and academic performance of MBBS students in pharmacology - A retrospective cohort study. National J Physiol, Pharm Pharmacol 2017;7(10):1056-1060. 